\title{
A Atenção Primária como parte integrante da rede de atendimento as Urgências e Emergências: à luz da literatura
}

\author{
Primary care as an integral part of the emergency care network: in the light of the literature \\ Atención primaria como parte integral de la red de atención de emergencia: a la luz de la \\ literatura
}

Taysa Cristina Cardoso Freitas ${ }^{1 *}$, Glenda Gicélia Ferreira Moreira ${ }^{1}$, Johnny Marques Aquino, Karla Priscila Cardoso Lacerda ${ }^{1}$, Reinaldo da Silva ${ }^{1}$, Amanda Poliana Goveia Araújo de Jesus ${ }^{1}$, Ana Cláudia Santos Fonseca', Karine Barbosa Gonçalves ${ }^{1}$, Aline Soares Botelho, Mirielle Dias Gonçalves'.

\begin{abstract}
RESUMO
Objetivo: Identificar a atuação dos profissionais da atenção primária a saúde frente as urgências e emergências à luz da revisão integrativa da literatura. Métodos: Trata-se de uma revisão integrativa da literatura cuja busca de publicações foi realizada nos meses de outubro e novembro de 2019 nas bases de dados Scientific Electronic Library Online (Scielo) e Biblioteca Virtual de Saúde (BVS), no corte temporal de 2013 a 2017. Resultados: Foram incluídos no estudo, 4 artigos que atenderam aos critérios de inclusão. A partir da análise dos artigos observou-se que o maior número de publicações em revistas de saúde publica, no ano de $2016 \mathrm{com}$ enfase em estudo descritivo exploratório de abordagem qualitativa. Considerações finais: Para que a atenção básica seja participante e atuante efetiva na rede de urgências, cabe aos gestores a inclusão deste tema em conferências municipais de saúde e treinamento para que toda a equipe possa prestar um atendimento efetivo ao usuário em situação de urgência e emergência.
\end{abstract}

Palavras-chave: Urgência, Emergência, Atenção Primária à Saúde.

\begin{abstract}
Objective: To identify the role of primary health care professionals in the face of urgencies and emergencies in light of the integrative literature review. Methods: This is an integrative literature review, which was searched for publications in October and November 2019 in the Scientific Electronic Library Online (Scielo) and Virtual Health Library (VHL) databases, in the 2013 temporal section. Results: Four articles that met the inclusion criteria were included in the study. From the analysis of the articles, it was observed that the largest number of publications in health journals published, in 2016, with emphasis on descriptive exploratory study. Final Considerations: For primary care to be a participant and effective in the emergency network, it is up to managers to include this theme in municipal health conferences and training so that the entire team can provide effective care to the user. in urgent and emergency situations.
\end{abstract}

Keywords: Urgency, Emergency, Primary Health Care.

\section{RESUMEN}

Objetivo: identificar el papel de los profesionales de atención primaria de salud frente a urgencias y emergencias a la luz de la revisión integral de la literatura. Métodos: Esta es una revisión bibliográfica integradora, que se buscó para publicaciones en octubre y noviembre de 2019 en las bases de datos de la Biblioteca Electrónica Científica en línea (Scielo) y de la Biblioteca Virtual en Salud (BVS), en la sección temporal de 2013. Resultados: se incluyeron en el estudio cuatro artículos que cumplían con los criterios de inclusión. Del análisis de los artículos, se observó que el mayor número de publicaciones en revistas de salud

\footnotetext{
${ }^{1}$ Faculdade de Saúde e Desenvolvimento Humano Santo Agostinho, Montes Claros - Minas Gerais.

*E-mail: cristina.cardoso.taysa@gmail.com
} 
publicaron, en 2016, con énfasis en el estudio exploratorio descriptivo. Consideraciones finales: Para que la atención primaria participe y sea efectiva en la red de emergencias, corresponde a los gerentes incluir este tema en las conferencias y capacitación en salud municipal para que todo el equipo pueda brindar una atención efectiva al usuario. en situaciones urgentes y de emergencia.

Palabras-clave: Urgencia, Emergencia, Atención Primaria de Salud.

\section{INTRODUÇÃO}

A Atenção Primária, no âmbito legislativo do Sistema Único de Saúde, está organizada como porta de entrada preferencial ao sistema. Na concepção desse nível assistencial, o cuidado centrado no usuário deve responder a todas as necessidades de saúde da população por meio de um sistema integrado, articulado e pautado na construção de redes de trabalho intersetoriais (BARBOSA MAF, et al., 2011).

A Unidade Básica de Saúde (UBS) tem seu campo de trabalho considerado de baixa densidade e de alta complexidade, lidando diariamente com uma grande variedade de problemas, desde aqueles gerados pelas mazelas sociais que requerem um trabalho intersetorial para a construção de respostas, até aquelas situações clínicas que demandam ações nas quais a própria equipe de saúde deve articular se para responder de forma resolutiva (LUMER S e RODRIGUES PHA,2011).

No dia a dia, a UBS busca através de uma amplitude de abordagens, soluções as diferentes situações de doenças e agravos à saúde. Entretanto, muitas vezes se depara com alguns casos mais complexos que exigem atendimento imediato, como é o caso das urgências e emergências (GARCIA AB, et al.,2012) .

Predominantemente, essas demandas são atendidas nos serviços hospitalares ou nas unidades de pronto atendimento. Entretanto, há situações em que o indivíduo que sofre determinado agravo, de natureza clínica, traumática ou psiquiátrica, procura por socorro no local mais próximo a sua residência, ou seja, a Estratégia Saúde da Família do seu bairro (BARBOSA MAF, et al., 2011).

A Portaria de ํㅜ 2.048, estabelecida pelo Ministério da Saúde em 5 de novembro de 2002, preconiza que os diferentes níveis de atenção devem se relacionar através de mecanismos de referência e contra referência, de modo que as ações possam ser complementares entre os níveis assistenciais e que cada serviço possa prestar seu atendimento à população conforme sua especialidade, realizando o encaminhamento para os outros níveis quando não possuir insumos suficientes para prestar este atendimento (BRASIL MS, 2002).

Além disso, prevê que as Unidades estejam organizadas com área física e materiais adequados para os atendimentos de urgência, como, também, amparadas com fluxos e protocolos de encaminhamentos para os demais níveis de complexidade (BRASIL MS, 2002). Em detrimento dessa necessidade, a portaria discorre a respeito da formação insuficiente e o despreparo dos profissionais da Atenção Primária à Saúde para atuar frente às urgências, ressaltando a importância e a garantia de qualificação para esse enfrentamento, com vistas à efetividade da assistência prestada (BRASIL, 2002).

O documento Humaniza SUS, elaborado pelo Ministério da Saúde (2004) afirma que os profissionais da atenção básica tem dificuldades de prestar atendimento ao paciente que chega à unidade com uma urgência de maior gravidade. $O$ impulso de encaminhar o paciente rapidamente à um nível assistencial mais complexo sem realizar sequer uma avaliação e estabilização do paciente pode estar relacionado à falta de capacitação profissional para este tipo de atendimento (BRASIL MS, 2004).

Atualmente, tem-se como subsidio legal que a organização das redes de atendimento às urgências e emergências abrange o componente pré hospitalar fixo e móvel, que abriga as Unidades Básicas de Saúde (UBS), Estratégias Saúde da Família (ESF) e o Serviço de Atendimento Móvel de Urgência (SAMU) além do sistema inter e pós-hospitalar (BRASIL MS, 2009).

Desse modo, as unidades de saúde da família têm papel de suma importância na rede de atendimento as urgências e emergências, devendo prestar o atendimento através de profissionais competentes, qualificados e possuir recursos físicos e materiais adequados para o devido atendimento. 
Considerando a necessidade de capacitação dos profissionais para 0 atendimento de agravos emergenciais na Atenção Primária à Saúde, o presente estudo tem como objetivo identificar através de uma revisão integrativa da literatura a atuação dos profissionais da atenção primária a saúde frente as urgências e emergências.

\section{MÉTODOS}

O presente estudo caracteriza-se como uma revisão integrativa da literatura realizada no período de novembro a dezembro de 2019. Para a revisão, definiu se as palavras chave a partir da plataforma de Descritores em Ciências da Saúde (DECS) a saber: Urgência, Emergência e Atenção Primária à Saúde. Posteriormente realizou-se o cruzamento destes descritores associados ao operador lógico de pesquisa AND, que tem a função de relacionar as palavras no processo de elaboração da pesquisa na Biblioteca Virtual em Saúde (BVS SALUD) (MENDES EV, 2011).

O levantamento do material a ser analisado foi realizado através de busca por artigos publicados no período de 2013 a 2017, em periódicos científicos nacionais disponíveis na Biblioteca Virtual em Saúde (BVS) e Scientific Electronic Library Online (SCIELO), que apresentam periódicos nacionais com artigos pertinentes ao tema. De acordo com os critérios de inclusão, os artigos deveriam ser originais, estar disponíveis para leitura na íntegra, voltados ao objetivo de estudo, com resumos disponíveis nas bases de dados selecionadas e publicados entre 2013 a 2017. Quanto aos critérios de exclusão: artigos publicados em período que antecede $o$ ano de 2013 e aqueles que apesar de apresentarem os descritores selecionados não abordem diretamente o tema proposto da pesquisa.

\section{RESULTADOS}

Figura 1 - "Urgência AND Atenção Primária a Saúde".
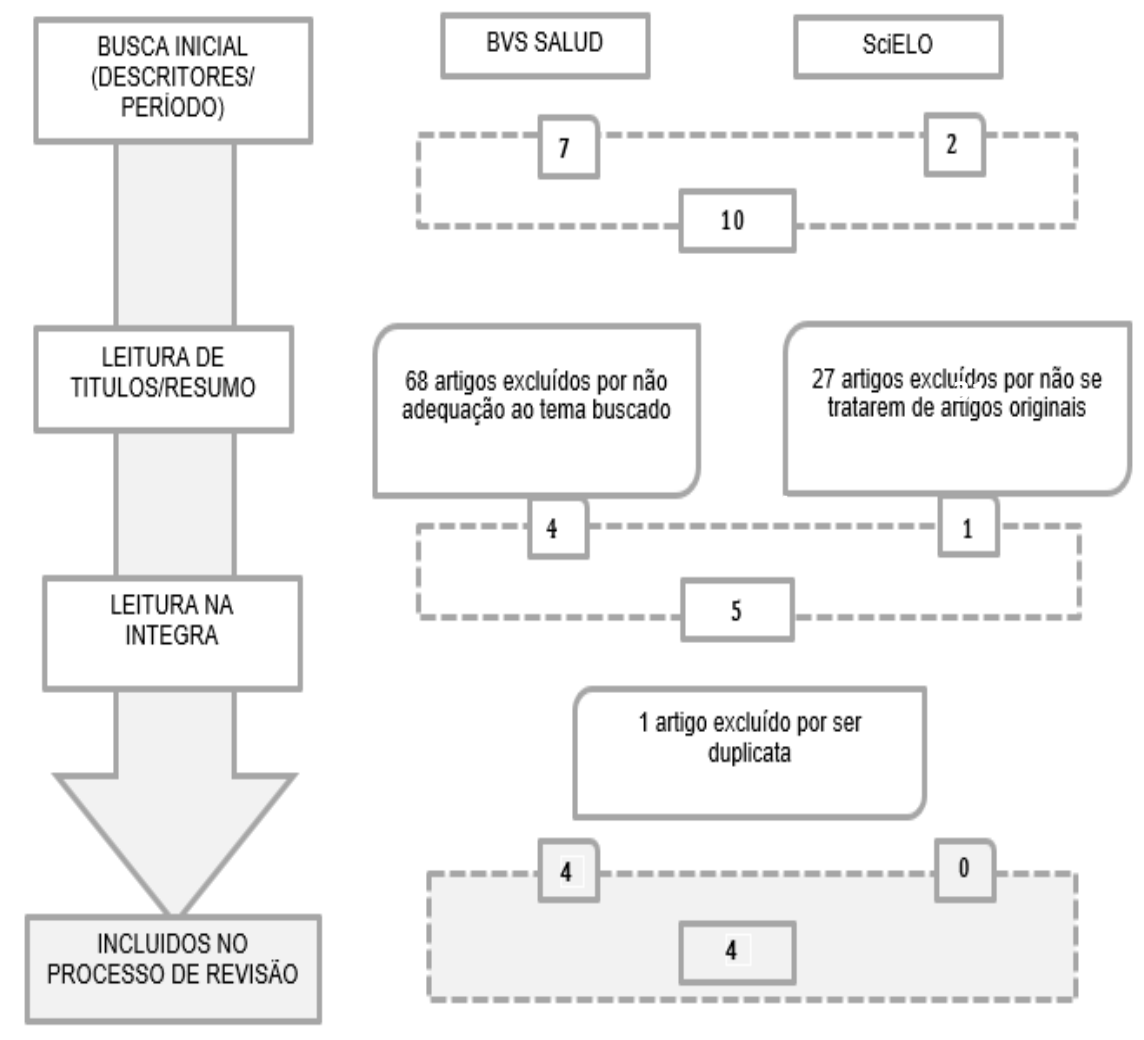

Fonte: Freitas TCC et al., 2020. 
A partir do cruzamento dos descritores e aplicação dos critérios de inclusão foram selecionados inicialmente 100 artigos. Após a leitura inicial de títulos e resumos foram excluídos 95 artigos, em seguida, as publicações foram pré-selecionadas com base no tipo de estudo e na leitura do texto na integra. Após a análise final um artigo foi excluído por se tratar de duplicata, nesse contexto, foi possível selecionar 4 artigos com potencial para compor a amostra final conforme apresentado na (Figura 1).

Após a seleção dos estudos que fariam parte do corpo de revisão, os mesmos foram submetidos a análise minuciosa para a extração dos tópicos essenciais do estudo e, de acordo com seus objetivos, foram comparados e agrupados em categorias tendo como referência a análise de conteúdo mediante correlação com a revisão de literatura utilizada, através dos passos de: ordenação de dados, classificação dos dados e análise interpretativa (MINAYO MCS, et al., 2015).

A fim de analisar de forma integral as informações dos artigos, eles tiveram seus principais tópicos plotados em um instrumento que favoreceu a estratificação dos estudos. Por meio da análise de conteúdo foram elaboradas duas grandes categorias, a saber: (1) A atuação da atenção primaria a saúde no acolhimento e referência ao paciente agudo, (2) O profissional de saúde da atenção primaria frente ao paciente agudo (Tabelas 1 e 2).

Tabela 1 - Distribuição das bibliografias potenciais encontradas nas bases de dados segundo a categoria (1): A atuação da atenção primaria a saúde no acolhimento e referência ao paciente agudo.

\begin{tabular}{lll}
\hline \multicolumn{1}{c|}{ Autor-Data } & Delineamento do Estudo & \multicolumn{1}{c}{ Objetivos } \\
\hline Gomes GG (2013) & $\begin{array}{l}\text { Tratiar a conduta dos profissionais da atenção } \\
\text { caso de natureza qualitativa. }\end{array}$ & $\begin{array}{l}\text { Entender as percepções dos profissionais quanto à } \\
\text { responsabilidade da UBS na rede de atenção às } \\
\text { urgências emergências. Verificar o fluxo de } \\
\text { atendimento disponibilizado aos pacientes que } \\
\text { chegam na Unidade básica de saúde com casos } \\
\text { agudos e os encaminhamentos destes para os níveis } \\
\text { mais complexos da assistência. }\end{array}$ \\
\hline Farias DC, et al., Pesquisa exploratória, \\
(2014)
\end{tabular}

Fonte: Freitas TCC, et al., 2020.

Tabela 2 - Distribuição das bibliografias potenciais encontradas nas bases de dados segundo a categoria (2): O profissional de saúde da atenção primaria frente ao paciente agudo.

\begin{tabular}{|c|c|c|}
\hline Autor-Data & $\begin{array}{l}\text { Delineamento do } \\
\text { Estudo }\end{array}$ & Objetivos \\
\hline $\begin{array}{l}\text { Oliveira TA, et al., } \\
2016\end{array}$ & $\begin{array}{l}\text { Estudo descritivo, com } \\
\text { abordagem qualitativa }\end{array}$ & $\begin{array}{l}\text { Analisar a percepção dos profissionais da Estratégia } \\
\text { Saúde da Família sobre o atendimento de urgência e } \\
\text { emergência. }\end{array}$ \\
\hline $\begin{array}{l}\text { Hermida PMV, et } \\
\text { al., } 2016\end{array}$ & $\begin{array}{l}\text { Estudo descritivo- } \\
\text { exploratório e qualitativo }\end{array}$ & $\begin{array}{l}\text { Conhecer a percepção de equipes de Saúde da Família } \\
\text { sobre a atenção básica na Rede de Urgência. }\end{array}$ \\
\hline
\end{tabular}

Fonte: Freitas TCC, et al., 2020

REAS/EJCH | Vol.Sup.n.38 | e2881 | DOI: https://doi.org/10.25248/reas.e2881.2020 Página 4 de 9 
Dentre os artigos que respondiam a questão norteadora deste estudo, o maior número de publicações foi realizada no ano de $2016 \mathrm{com} \mathrm{50 \%} \mathrm{(n=2)} \mathrm{dos} \mathrm{artigos.} \mathrm{As} \mathrm{publicações} \mathrm{predominaram} \mathrm{nas} \mathrm{revistas} \mathrm{saúde}$ publica com enfase em estudo descritivo, de abordagem exploratória e qualitativa uma vez que essa abordagem permite conhecer a percepção da população estudada a respeito do tema proposto.

\section{DISCUSSÃO}

\section{A atuação da atenção primaria a saúde no acolhimento e referência ao paciente agudo}

A partir da publicação da portaria 4.279/2010 os serviços de Atenção Primária a Saúde (APS) passaram a ser reconhecidos como porta de entrada principal para os usuários do sistema Público de Saúde, tendo a função primordial de ordenar e coordenar o referenciamento do usuário aos devidos níveis de assistência à saúde demandadas pelo mesmo (BRASIL MS, 2010).

Para exercer essa função com excelência, ainda existem alguns desafios que precisam ser sanados na esfera de gestão da APS como referência universal ao usuário. No que se refere às demandas programadas, agendamentos, demanda espontânea e, especialmente, nos "casos agudos" que representam as situações nas quais os usuários chegam ao serviço apresentando desestabilização de quadros mórbidos pré-existentes ou situações de novos agravos à saúde, ainda existe uma serie de divergências que dificultam a prestação de uma assistência eficaz ao paciente.

Segundo Gomes GG (2013), estes usuários necessitam de atendimento rápido e eficiente com o objetivo de evitar complicações desnecessárias preferencialmente na Unidade Básica de Saúde de seu território adscrito. A organização da demanda nos serviços de APS, ainda que seja centrada em atendimentos programados não deve eliminar a necessidade e importância do atendimento à demanda espontânea por casos agudos (GOMES GG, 2013; RAMOS RR, 2009).

A resolução 1.451 de 10/03/1995, emitida pelo Conselho Federal de Medicina preconiza que a situação urgente é caracterizada pela "ocorrência imprevista de agravo à saúde com ou sem risco potencial de vida, cujo portador necessita de assistência médica imediata". O mesmo texto ainda discorre que emergência é a "constatação médica de condições de agravo à saúde que impliquem em risco iminente de vida ou sofrimento intenso exigindo, portanto, atendimento médico imediato" (CFM, 1995).

Gomes GG (2013) afirma que os usuários com casos de urgências e emergências, geralmente procuram a ESF por demanda espontânea, já com quadros agudizados de sofrimento físico, mental ou emocional e podem necessitar de assistência instantânea.

O atendimento desses casos demanda insumos que nem sempre estão disponíveis, tais como: insumos físicos, que caracterizam as tecnologias apropriadas para prestação do cuidado integral, medicamentos, estrutura, além de recursos de pessoal e reorganização da agenda do serviço (GOMES GG, 2013).

Muitas vezes os usuários que apresentam casos agudos são encaminhados para os níveis secundários ou terciários de assistência, estes por sua vez julgam não ser necessária sua intervenção, pois conforme a legislação que rege a rede de atendimento às Urgências e emergências, o usuário com quadro agudo poderia ser atendido na Unidade Básica de Saúde. Essas divergências geram conflitos na organização da assistência e prejudicam o paciente, que por sua vez, fica confuso quanto a representatividade da Atenção primária como referência para o cuidado de sua saúde (GOMES GG, 2013).

Ainda que o atendimento das urgências e emergências seja atribuído a todos os níveis da assistência conforme os recursos disponíveis, sabe-se que isso não ocorre de fato. Em decorrência de recursos estruturais, tecnológicos e mão de obra precários, o paciente acaba sendo o maior prejudicado nessas situações.

O Ministério da Saúde estabeleceu através da portaria no2048 de 2002 que uma retaguarda pactuada que permita o encaminhamento de pacientes acolhidos pela UBS e que necessitam de cuidados em níveis assistenciais mais complexos é de suma importância de suma importância .Além disso, a portaria preconiza 
que as unidades devem possuir um serviço de transporte para os casos agudos que chegam na unidade e precisam ser atendidos em outros pontos de atenção, esse tipo de transporte é realizado atualmente pelo serviço pré hospitalar móvel (SAMU) (BRASIL, 2002) .

Segundo Farias DC, et al. (2014), as equipes de saúde da família são multidisciplinares justamente por serem responsáveis por um atendimento integral aos seus usuários, incluindo dessa forma o atendimento às urgências, conforme o rol de deveres constantes na Política Nacional de Atenção Básica. Essa política preconiza que ao receber casos de urgência na unidade, a equipe de saúde deve realizar a correta classificação de risco, avaliação da situação de saúde e nível de vulnerabilidade do usuário, prestando o primeiro atendimento e encaminhando o paciente para o nível secundário de atenção caso seja necessário.

Corroborando com o estudo de Gomes GG (2013), Farias DC, et al., (2014) elenca através de seu estudo que, compreender o funcionamento da rede de serviços de saúde é essencial para a prestação de um atendimento realmente resolutivo, e esse entendimento deve ser transmitido a todos os profissionais, para que o usuário possa ter suas necessidades de saúde resolvidas no devido nível assistencial com agilidade.

Estudo realizado no ano de 2014 com profissionais da atenção primária a saúde do município de Campina Grande (PB), buscou verificar o fluxo de atendimento disponibilizado aos pacientes que chegam na Unidade básica de saúde com casos agudos. A pesquisa revelou que ainda existem inúmeros entraves para a adequada atenção as situações de urgência e emergência na Atenção primária a saúde, o que compromete o princípio de integralidade a saúde (FARIAS DC, et al., 2015).

Destaca-se principalmente a pouca capacitação das equipes multiprofissionais, déficit de insumos e medicamentos básicos para os atendimentos de urgência, estruturas insuficientes no que tange ao acolhimento e observação destes pacientes (GOMES GG,2013; TORRES AAP; SANTANA BP,2011).

O estudo também demonstra que os profissionais atuantes na estratégia saúde da família apresentam dificuldades em reconhecer as situações de urgência e muitos deles, desconhecendo os aspectos legais, não consideram de sua responsabilidade esse tipo de atendimento. Desse modo, os profissionais utilizam o encaminhamento como única alternativa de resolubilidade para os casos agudos (FARIAS DC, et al., 2015).

Estudo realizado com 16 profissionais de uma ESF concluiu que maiores dificuldades dos profissionais estão relacionadas a falta de educação permanente. A política Nacional de atenção às Urgências e Emergências é pouco conhecida e divulgada no contexto da UBS (NOBREGA DM, et al., 2015).

Para sanar essas fragilidades é necessário aliar a prática e a teoria nos conceitos chave do sistema de Saúde, os principios de equidade, integralidade, universalidade e porta de acesso que destacam a atenção primária, tem como elo principal a educação. A gestão da APS precisa ser repensada com o objetivo de garantir ao usuário assistencia integral independente do seu quadro de saúde, fortalecendo assim o vinculo Usuário- APS (SANTOS AP, et al., 2018).

Desse modo, a presente categoria nos permitiu compreender que ainda existem inúmeros desafios a serem sanados no que tange 0 atendimento das urgências e emergências na Atenção primária a Saúde, destaca-se a importância da capacitação dos profissionais para uma prestação de assistência adequada e eficiente, além da importância da interligação entre os níveis assistenciais para que a referência e contrareferencia possam funcionar.

\section{O profissional de saúde da atenção primaria frente ao paciente agudo}

A atenção básica está inserida na rede de atenção às situações de urgência como um componente de ampliação do acesso, desde os cuidados primários até a transferência do usuário a outros níveis de atenção quando necessário (BRASIL MS, 2003).

Os profissionais atuantes na equipe multidisciplinar de saúde precisam estar prontos para prestar o atendimento aos pacientes com quadros agudos em seu território, uma vez que a história clinica já é conhecida, possibilitando a readequação terapêutica dentro da disponibilidade da unidade de saúde (BRASIL, 2011). 
Estudo realizado no ano de 2013 com 70 profissionais da atenção primária a saúde procurou analisar a percepção dos profissionais da Estratégia Saúde da Família sobre o atendimento de urgência e emergência. A pesquisa evidenciou que os profissionais atuantes na ESF percebem a atenção primária como uma estratégia baseada em ações de promoção e prevenção e proteção da saúde realizando grupos operativos, visitas domiciliares e consultas ambulatoriais com vistas a cumprir as metas dos programas de atenção à saúde (OLIVEIRA TA, et al., 2016).

Infelizmente muitos profissionais atuantes na atenção primária desconhecem a estratégia saúde da família como integrante da rede de atenção as urgências e emergências, bem como as legislações relacionadas a este tema. Este fator elenca a necessidade constante de educação permanente para todos os membros da equipe multiprofissional. A educação Permanente tem o poder de transformar as práticas e organização do trabalho em saúde. Entretanto, o acumulo de conhecimento técnico cientifico é apenas um dos aspectos para a transformação das práticas diárias na UBS (SAMPAIO GB, et al., 2019).

Em um estudo realizado com 20 profissionais atuantes em três Centros de Saúde do município de Palhoça, em Santa Catarina, investigou-se as percepções das equipes de saúde da Família sobre a atenção básica inserida na rede de atendimento às urgências. Segundo o estudo, os profissionais atendem às situações de urgência e emergência por compreenderem ser a unidade referência e porta de entrada para a população com demandas de saúde urgentes ou não, após o atendimento inicial, providenciam o encaminhamento do usuário para unidades de maior porte (HERMIDA PMV, et al., 2016).

Enfim, Hermida PMV, et al., (2016) concluíram que existem muitas fragilidades no processo de assistência ao paciente agudo, a grande maioria delas estão associadas à estrutura física precária, aos recursos materiais escassos e à qualificação profissional para a estabilização dos pacientes e, como aspecto potencial, a acessibilidade do usuário ao serviço, o comprometimento da equipe no atendimento e o reconhecimento de que a atenção básica compõe a rede de atenção às urgências, contribuindo para diminuir a demanda nos serviços de emergência hospitalar.

Para sanar estas dificuldades, Gomes GG (2013) discorre que nas situações de urgência e emergência na atenção primária , a existência de fluxos e protocolos são ferramentas de suma importância na tomada de decisão, auxiliando os profissionais a aplicarem a pratica assistencial com segurança. Ao ofertar atendimento ao usuário agudo é importante que os possiveis desfechos do quadro possam ser previstos com vistas a preservar a saúde deste paciente.

A adoção de protocolos de triagem e acolhimento facilitam a sistematização da assistencia, permitindo a inserção dos usuários e a otimização da prestação do cuidado. Além disso, através deles é possivel hierarquizar os riscos de modo que os profissionais consigam, desde a entrada do paciente na UBS, saber qual a demanda de recursos necessária e quais os niveis assistenciais o usuário deve ser encaminhado (OLIVEIRA M e TRINDADE MF,2017)

Oliveira M e Trindade MF (2017) afirmam que quando a prática está aliada a um plano assistencial sistemico, as intervenções de saúde garantem um atendimento rápido e efetivo, o que é essencial quando o paciente a ser atendido apresenta um quadro agudo. Um bom diagnóstico e a rápida abordagem dos sinais de piora são de suma importância num prognóstico bem-sucedido. Por esse e outros motivos, destaca-se que os profissionais precisam estar capacitados para a realização desse acolhimento.

Os estudos incluídos nesta categoria revelam a necessidade de reformulações na atenção primária para que os usuários em situação de urgência e emergência sejam assistidos com qualidade e eficiência, sendo encaminhados adequadamente aos outros níveis assistenciais quando necessário. Destaca-se que a implantação de sistemas de informação, protocolos e fluxogramas não conseguem alcançar resultados satisfatórios se a equipe de saúde não consegue atuar em sintonia com as necessidades do usuário e nem com a demanda de serviço que lhe é atribuída.

Por se tratar de uma revisão integrativa com corte temporal, a escassa quantidade de estudos pode caracterizar-se como uma limitação. Entretanto, em nossas buscas foi possível observar a pequena 
quantidade de estudos relacionados com o tema em questão, gerando assim uma lacuna do conhecimento que precisa ser preenchida. Desse modo, destacamos a extrema importância de novos trabalhos que busquem analisar os a atenção primária e os profissionais ali atuantes no tocante a sua atuação frente às situações de urgência e emergência que chegam na unidade básica de Saúde.

\section{CONSIDERAÇÕES FINAIS}

Através de nosso estudo foi possível concluir que cabe aos gestores da atenção primária incentivar seus profissionais a colocarem em prática as portarias estabelecidas pelo Ministério da Saúde. Em destaque a portaria 1600/2011, para que a atenção básica seja participante e atuante efetiva na rede de urgências, além disso destaca-se que os profissionais atuantes nas unidades precisam ter ciência e capacitação continua para que possam prestar o cuidado de modo eficiente.

\section{REFERÊNCIAS}

1. BARBOSA MAF , et al. Capacitação dos profissionais de saúde para o atendimento de parada cardiorrespiratória na atenção primária. Revista de Atenção Primária a Saude, 2011;14 (2):233-238.

2. BRASIL MS. Regulamento Técnico dos Sistemas Estaduais de Urgência

3. e Emergência. Portaria GM/MS no 2.048, de 5 de Nov. de 2002. . Diário Oficial da União,Brasília, 2002.

4. BRASIL MS. Portaria GM/MS no. 1.863, de 29 de setembro de 2003.Institui a Política Nacional de Atenção às Urgências, a ser implantada em todas as unidades

5. federadas, respeitadas as competências das três esferas de gestão. . Diário Oficial da União,Brasília, 2003.

6. BRASIL MS. Humaniza SUS:acolhimento com avaliação e classificação de risco: um paradigma ético- estético no fazer em saúde. Brasília: Ministério da Saúde; . 2004.

7. BRASIL MS. Portaria GM/MS no. 1.020, de 13 de maio de 2009. Estabelece diretrizes para implantação do componente pré- hospitalar fixo para a organização de redes locorregionais de atenção integral às urgências em conformidade com a Política Nacional de Atenção às Urgências.

8. . Diário Oficial da União, Brasília, 2009.

9. BRASIL MS. Portaria oㅜ 4279, de 30 de dezembro de 2010. Estabelece diretrizes para a organização da Rede de Atenção à Saúde no âmbito do Sistema Único de Saúde(SUS). . Diário Oficial da União, Brasília (DF) 2010.

10. BRASIL MS. Decreto № 7.508, de 28 de junho de 2011. Regulamenta a lei 8.080, de 19 de setembro de 1990, para dispor sobre a organização do Sistema Único de Saúde- SUS, o planejamento da saúde, a assistência à saúde e a articulação interfederativa e dá outras providências. . Diário Oficial da União, Brasília, 2011.

11. CFM CFM. Resolução 1.451, de 10 de março de 1995. Dispõe sobre o funcionamento de hospitais públicos e privados. . Diário Oficial da União, Brasília, : 10 março 1995. 1995.

12. DE OLIVEIRA TA, et al. Percepção de profissionais da estratégia saúde da família sobre o Atendimento de urgência e emergência. Journal of Nursing UFPE/Revista de Enfermagem UFPE, 2016;10 (3):1397-1406.

13. FARIAS DCd, et al. Acolhimento e resolubilidade das urgências na estratégia saúde da família. Revista Brasileira de Educação Médica, 2015;39 (1):79-87.

14. GARCIA AB , et al. Estratégia da Saúde da Família: capacidade da equipe para 0 atendi-mento de urgência e emergência. Revista Nursing, 2012;14 (167):216-220.

15. GOMES GG. Atendimento de usuários com casos agudos na atenção primária à saúde. (Dissertação de Mestrado) Dissertação de Mestrado, Universidade Federal de Minas Gerais, Belo Horizonte: .2013,89.

16. HERMIDA VIEIRA PM, et al. Percepção de equipes de saúde da família sobre a atenção básica na rede de urgência. Journal of Nursing UFPE/Revista de Enfermagem UFPE, 2016;10 (4):1170-1178.

17. LUMER S; RODRIGUES, PHdA. O papel da saúde da família na atenção às urgências. Revista de Atenção Primária a Saude, 2011;14 (3):289-295.

18. MENDES EV. Os modelos de atenção à saúde. As Redes de Atenção à Saúde. Brasília: Organização Pan-Americana da Saúde, 2011;1 (1):244-292.

19. MINAYO MCdS, et al. Pesquisa social: teoria, método e criatividade. In: Pesquisa social: teoria, método e criatividade, 2015. p. 80.

20. NÓBREGA DM, et al. Conhecimentos, atitudes e práticas em urgência e emergência na atenção primária à saúde. Ciência \& Desenvolvimento-Revista Eletrônica da FAINOR, 2015;8 (2):141-157.

21. OLIVEIRA M; TRINDADE, MF. Atendimento de urgência e emergência na rede de atenção básica de saúde: análise do papel do enfermeiro e o processo de acolhimento. HÓRUS, 2017;5 (2):160-171. 
22. RAMOS RR. A recepção da demanda espontânea no setor saúde da criança numa unidade básica de saúde. Dissertação de Mestrado (Dissertação de Mestrado) -, Universidade de São Paulo2009,79.

23. SAMPAIO GB , et al. Educação Permanente e o Processo Formativo dos Profissionais da Saúde: Entrelace de Saberes. Revista Eletrônica Acervo Saúde, 2019;1 (25):1-8.

24. SANTOS AAd, et al. O papel do enfermeiro como educador na atenção primária á saúde: uma revisão bibliográfica. Revista Eletrônica Acervo Saúde, 2018;2178 (11):1319-1324.

25. TORRES AAP; SANTANA, BP. Enfrentamento das emergências pelos profissionais da Estratégia de Saúde da Família. Journal of Nursing and Health, 2011;1 (1):107-112. 